Fluorescence in situ hybridization study that revealed a deletion on chromosome 22q11.2 zone. Therapy with calcium was undertaken at first by intravenous infusion and orally afterwards; high levels of calcium were needed to normalize serum calcium.

Molecular evaluation of the parents showed no deletion in the $22 q 11.2$ zone, allowing for the diagnosis of a de novo deletion in the index case.

The importance of this report relies on the fact that the patient, despite clinical suspicions of VCFS, remained asymptomatic until late childhood, presenting with no renal dysfunction, immunological abnormalities or cardiac malformations.

Considering this hypothesis and making an early diagnosis is important both for implementing timely clinical evaluation and dietary supplementation if needed and for family planning.

\section{DIFFERENT FACES OF CHRONIC AUTOIMMUNE THYROIDITIS IN CHILDHOOD AND ADOLESCENCE}

doi:10.1136/archdischild-2012-302724.0664

L Saranac. Pediatric Clinic, University Clinical Centre Nis, Nis, Serbia

Chronic autoimmune thyroiditis (CAT) is multifaced disease. Its incidence has increased dramatically over the past few decades afflicting up to $2 \%$ of the general population. CAT as autoimmune disorder results from complex interplay of genetic, environmental and endogenous factors. In nonendemic goitre areas, CAT is the most common cause of acquired hyothyroidism.

Unlike overt goitrogenic form of disease, atrophic one remains hidden or misdiagnosed for years.

Some children expirience all thyroid dysfunction types during natural course of the disease; mild hyperthyroidism at diagnosis (hashitoxicosis), euthyroid state, and gradual progression from subclinical to overt hypothyroidism.

The clinical manifestations of acquired hypothyroidism (AH) in childhood differ from those in adults. The classic manifestation also occur in children, but are not so prominent. Instead, the most important sign of $\mathrm{AH}$ in childhood is growth failure. Weight tends to increase and in most instances weight for age is greater than height for age. The retardation of bone age in AH usually equals or exceeds the retardation in linear growth. Pubertal disorders: delayed, or even precocious puberty are always present.

Another intriguing form of dysfunction in CAT could be the subclinical hypothyroidism with mixed signs of hypo and hyperfunction (autoimmune dysthyroidism). Thus, clinical features do not always correspond to hormonal status. The reasons for diagnostic pitfalls, because of clinical ambiguity are challenging for pediatricians and endocrinologists.

\section{RETROPHARYNGEAL ABSCESS AS A PITFALL IN THE DIAGNOSIS OF KAWASAKI DISEASE: CASE REPORT AND REVIEW OF THE LITERATURE}

doi:10.1136/archdischild-2012-302724.0665

${ }^{1} \mathrm{~L}$ Da Dalt, ${ }^{1} \mathrm{P}$ Berlese, ${ }^{1} \mathrm{ME}$ Cavicchiolo, 'MS Strafella, ${ }^{2} \mathrm{E}$ Trincia, ${ }^{2}$ I Inches, ${ }^{3} \mathrm{~S}$ Bressan. 'Pediatric Unit; ${ }^{2}$ Neuroradiology Unit, Treviso Hospital, Treviso; ${ }^{3}$ Department of Pediatrics, University of Padova, Padova, Italy

Kawasaki disease $(\mathrm{KD})$ is an acute self-limiting vasculitis of childhood of unknown etiology. We report the case of a patient with $\mathrm{KD}$ whose initial presentation mimicked a retropharyngeal abscess (RA) and review the literature on this topic. Our child, a 4 year old girl, presented with fever ( $<24$ hours) and clinical, laboratory and MRI findings.

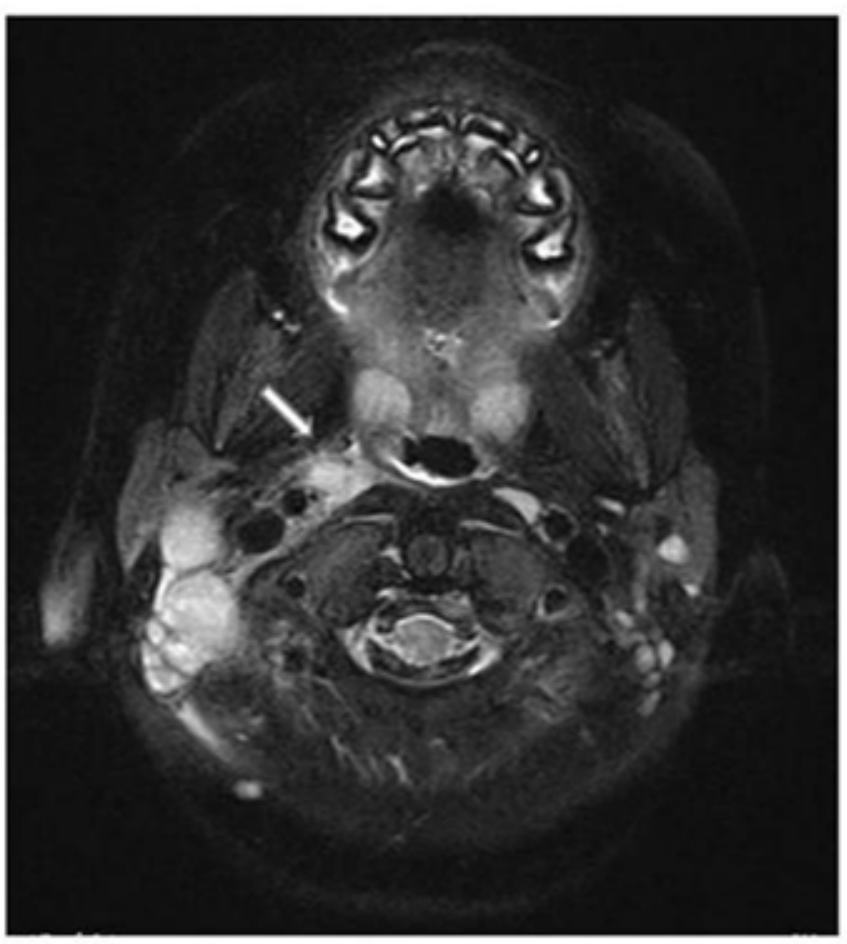

Abstract 665 Figure 1

Consistent with RA. She received prompt intravenous antibiotic therapy with no clinical improvement. Only repeated accurate physical examinations, with early appreciation of subsequent clinical findings consistent with $\mathrm{KD}$, allowed for early diagnosis and proper treatment with intravenous immunoglobulin. In literature sixteen cases of $\mathrm{KD}$ mimicking RA have been reported. Fever and deep neck infection like symptoms were the only clinical findings at admission in 14 (87.5\%) children. All children had a neck CT scan performed showing findings suggestive of RA. All children were promptly started intravenous antibiotic therapy without clinical improvement and five patients (31\%) underwent unproductive surgical drainage of the retropharyngeal space. Twelve patients $(80 \%)$ received a diagnosis of $\mathrm{KD}$ after 7 or more days since onset of fever and 4 after 10 or more days. Coronary aneurisms were found in 2 patients, both of whom had a delayed KD diagnosis after 10 or more days since fever onset.

Conclusion Pediatricians should be aware of atypical KD presentations mimicking RA. Early diagnosis of $\mathrm{KD}$ is pivotal for preventing cardiac complications, as well as avoiding the risk associated to unnecessary surgical intervention.

\section{PULMONARY ABNORMALITIES IN CHILDREN AND ADOLESCENTS WITH TYPE 1 DIABETES MELLITUS}

doi:10.1136/archdischild-2012-302724.0666

${ }^{1} \mathrm{GC}$ Yılmaz, 'P Boran, '2L Yılmaz, 'E Tutar, 'G Tokuc. 'Dr. Lutfi Kirdar Kartal Research and Training Hospital, 2nd Clinic of Pediatrics; ${ }^{2}$ Dr. Lutfi Kirdar Kartal Research and Training Hospital, Department of Family Medicine, Istanbul, Turkey

Background and Aims Few studies are available on pulmonary function abnormalities in children with diabetes with controversial results. Spirometric abnormalities and reduction of lung diffusing capacity for carbon monoxide (DLCO) have been reported.

A cross sectional study was designed to assess whether children and adolescents with type 1 diabetes have pulmonary dysfunction. 